\title{
Peertechz
}

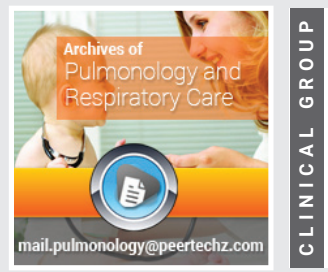

\section{A Two-year review of the use of film array in a tertiary hospital in the Philippines}

\section{Evelina N Lagamayo and Ruby 0 Rusia-Uy*}

Department of Pathology, Vicente Sotto Memorial Medical Center, B. Rodriguez Street, Cebu City, 6000

Cebu, Philippines
Received: 18 January, 2021

Accepted: 02 February, 2021

Published: 03 February, 2021

*Corresponding author: Ruby O Rusia-Uy, Department of Pathology, Vicente Sotto Memorial Medical Center, B. Rodriguez Street, Cebu City, 6000 Cebu, Philippines, E-mail: ubings@yahoo.com

Keywords: Multiplex polymerase chain reaction; Gastrointestinal panel; Respiratory panel; Meningitis encephalitis panel; Blood culture identification panel

https://www.peertechz.com

Check for updates

\section{Abstract}

Background: Molecular methods such as Multiplex Polymerase Chain Reaction (PCR) has the potential to rapidly identify pathogens for diagnosing infectious diseases.

Objective: This study aims to describe the usage of the Multiplex PCR System (FilmArray®) Gastrointestinal, Respiratory, Meningitis Encephalitis, and Blood Culture Identification Panels done from October 2017 to September 2019.

Methods: Results of all the panels done during the study period were retrieved from the laboratory database. Data were entered into the collection forms and analyzed to achieve the objectives.

Results: A total of 547 test results were gathered. The most requested panel among the four was Gastrointestinal Panel (48\%). Majority of the patients tested were from those aged 60 years old and above at $29.8 \%$. Patients who had the panels tested were mostly males (55\%) and mostly requested among inpatients at $69 \%$. The positivity rates of the Gastrointestinal, Respiratory, Meningitis Encephalitis Panel, and Blood Culture Identification panels were $60 \%, 57 \%, 14 \%$ and $83 \%$, respectively. All panels had both single detections as well as codetections except for Blood Culture Identification Panel which only yielded single pathogen. The most common requesting physicians are the Gastroenterologists (25.9\%) followed by Infectious Disease Specialists (15.9\%). Collectively, Pediatricians and other Pediatric Specialty Physicians comprise $19.5 \%$ of the requesting doctors.

Conclusion: The Multiplex PCR FilmArray Panels were useful both in the outpatient and inpatient care and a wide range of patients. The high positivity rates provide early patient diagnosis, thereby, early administration of appropriate targeted therapy and management especially those which might have been missed by the conventional culture. Identification of viral etiologies reduce the unnecessary use of antimicrobials and greatly help in the reduction of the development of antimicrobial resistance.

\section{Introduction}

Sepsis is a life-threatening organ dysfunction caused by a dysregulated host response to infection. If not recognized early and managed promptly, it can lead to septic shock, multiple organ failure and death. Any type of infectious pathogen can potentially cause sepsis. Antimicrobial resistance is a major factor determining clinical unresponsiveness to treatment and rapid evolution to sepsis and septic shock. Sepsis patients with resistant pathogens have been found to have a higher risk of hospital mortality [1]. By shortening the time to pathogen identification and allowing for detection of organisms missed by blood culture, new molecular methods may provide clinical benefits for the management of patients with sepsis
[2]. Antimicrobial Resistance (AMR) threatens the effective prevention and treatment of an ever-increasing range of infections caused by bacteria, parasites, viruses and fungi. Patients with infections caused by drug-resistant bacteria are at increased risk of worse clinical outcomes and death, and consume more health-care resources than patients infected with non-resistant strains of the same bacteria [3].

Current microbiological techniques are limited in their diagnostic capacities and timeliness. Multiplex Polymerase Chain Reaction (PCR) has the potential to rapidly identify bloodstream infections and fill this diagnostic gap [4]. Molecular methods for diagnosing infectious diseases have become part of routine testing algorithms in clinical laboratories of all sizes. Laboratory and clinical personnel are enthusiastic about 
this because molecular testing provides higher sensitivity and specificity and, in most cases, a shorter turnaround time than traditional methodologies. For example, while conventional culture and susceptibility testing may require 72 hours to produce results, multiplex PCR assays can do so in 1 to 3 hours after the blood culture is flagged as positive by the blood culture instrument. Benefits from this rapid testing will be realized if therapeutic decisions are made as soon as results are available; therefore, support from an antimicrobial stewardship team is paramount to achieve the goals of cost-effective testing and improved patient outcomes [5]. Detecting causative organism and ruling out infectious etiology remain the most critical aspect for management and prognosis of patients with suspected CNS infections. In a study by Javali, et al published in PubMed entitled, "Use of multiplex PCR based molecular diagnostics in diagnosis of suspected CNS infections in tertiary care setting-A retrospective study", it was observed that there is higher detection rate of pathogens, target specific escalation and evidence based de-escalation of antimicrobials using Syndrome Evaluation System (SES). Institution of appropriate therapy helped reduce unnecessary use of antimicrobials [6]. Given the advantages already demonstrated by the use of multiplex PCR along with the recent developments in this technology, future applications of PCR, when possible, should be aimed at constructing multiple detection systems in which a number of clinically and epidemiologically relevant pathogens (viruses, bacteria, parasites, and/or fungi) may be detected, characterized, and/or inevitably uncovered in a symptomand/or system-specific manner [7]. A study on the Preliminary experience with the use of Multiplex PCR System (FilmArray ${ }^{\circledR}$ ) in a hospital from Argentina concluded that point of care multiplex PCR panel test might be useful in the acute care of patients with unknown infectious disease syndromes and negative standard test methods [8]. In this study, the usage of Biofire ${ }^{\circledR}$ FilmArray ${ }^{\circledR}$ system will be described. It is approved for use by regulatory bodies for medical devices such as Food and Drug Administration (FDA), CE Marking for In Vitro Diagnostic (CE-IVD), and Therapeutic Goods Administration (TGA) certifying this multiplex PCR system that integrates sample preparation, amplification, detection, and analysis. This simple system requires just 2 minutes of hands-on time, with a total run time of about an hour to assist in better patient care [9].

The BIOFIRE ${ }^{\circledR} \quad$ FILMARRAY ${ }^{\circledR}$ system enables simultaneous testing for bacteria, viruses, yeast, parasites and/or antimicrobial resistant genes. It is designed to be used with comprehensive panels that each offer testing for sets of pathogens associated with some of today's most pressing healthcare challenges.

BIOFIRE ${ }^{\circledR}$ FILMARRAY ${ }^{\circledR}$ respiratory panel: Tests for a comprehensive panel of 20 respiratory viruses and bacteria namely Adenovirus, Coronavirus 229E, Coronavirus HKU1, Coronavirus OC43, Coronavirus NL63, Human Metapneumovirus, Human Rhinovirus/Enterovirus, Influenza A, Middle East Respiratory Syncytial CoronaVirus (Mers-CoV), Influenza A/H1, Influenza A/H12009, Influenza $\mathrm{A} / \mathrm{H}_{3}$, Influenza $\mathrm{B}$, Parainfluenza 1, Parainfluenza 2, Parainfluenza 3, Parainfluenza 4, Respiratory Syncytial Virus (RSV), Bordetella pertussis, Bordetella parapertussis, Chlamydophila pneumoniae, and Mycoplasma pneumonia.

\section{BIOFIRE ${ }^{\circledR}$ FILMARRAY ${ }^{\circledR}$ Blood Culture Identification}

Panel (BCID): Tests for a comprehensive list of 24 pathogens and 3 antibiotic resistance genes associated with bloodstream infections namely Enterococcus, Listeria monocytogenes, Staphylococcus, Staphylococcus aureus, Streptococcus, Streptococcus agalactiae, Streptococcus pyogenes, Streptococcus pneumoniae, Acinetobacter baumannii, Haemophilus influenzae, Neisseria meningitidis, Pseudomonas aeruginosa, Enterobacteriaceae, Enterobacter cloacae complex, Escherichia coli, Klebsiella oxytoca, Klebsiella pneumoniae, Proteus, Serratia marcescens, Candida albicans, Candida glabrata, Candida krusei, Candida parapsilosis, Candida tropicalis and the resistance genes mecA - methicillin resistance, vanA/B - vancomycin resistance, and KPC carbapenem resistance.

BIOFIRE ${ }^{\circledR}$ FILMARRAY ${ }^{\circledR}$ Gastrointestinal panel (GI): Tests for 22 common gastrointestinal pathogens including viruses, bacteria and protozoa that cause infectious diarrhea namely Campylobacter (jejuni, coli \& upsaliensis), Clostridium difficile (Toxin A/B), Plesiomonas shigelloides, Salmonella, Yersinia enterocolitica, Vibrio (parahaemolyticus, vulnificus, \& cholerae), Vibrio cholerae, E. coli 0157, Enteroaggregative E. coli (EAEC), Enteropathogenic E. coli (EPEC), Enterotoxigenic E. coli (ETEC) lt/st, Shiga-like toxin-producing E. coli (STEC) stx1/stx2 E. coli 0157, Shigella/Enteroinvasive E. coli (EIEC), Adenovirus F 40/41, Astrovirus, Norovirus GI/GII, Rotavirus A, Sapovirus (I,II, IV, and V), Cryptosporidium, Cyclospora cayetanensis, Entamoeba histolytica, and Giardia lamblia.

BIOFIRE ${ }^{\circledR}$ FILMARRAY ${ }^{\circledR}$ Meningitis Encephalitis panel (ME): Tests directly in Cerebral Spinal Fluid for the 14 most relevant ME-associated pathogens, including bacteria, viruses and a parasite namely Escherichia coli K1, Haemophilus influenzae, Listeria monocytogenes, Neisseria meningitidis, Streptococcus agalactiae, Streptococcus pneumoniae, Cytomegalovirus (CMV), Enterovirus, Herpes simplex virus 1 (HSV-1), Herpes simplex virus 2 (HSV-2), Human herpes virus 6 (HHV-6), Human parechovirus, Varicella zoster virus (VZV), and Cryptococcus neoformans/gattii.

BIOFIRE ${ }^{\circledR}$ FILMARRAY ${ }^{\circledR}$ performs the extraction, amplification and detection in a closed system, minimizing contamination. It is a syndromic testing system for a variety of pathogens associated with key healthcare concerns.The BIOFIRE ${ }^{\circledR}$ FILMARRAY ${ }^{\circledR}$ pouch stores all the necessary reagents for cell lysis, DNA/RNA extraction and purification, amplification, and detection in one closed pouch. BIOFIRE ${ }^{\circledR}$ FILMARRAY ${ }^{\circledR}$ extracts and purifies all nucleic acids from the unprocessed sample and performs a nested multiplex PCR. During PCR I, the system performs a single, large volume, multiplexed reaction. Individual, singleplex PCR II reactions detect the products from the first-stage PCR [10].

The FilmArray ${ }^{\circledR}$ menu comprises three panels, the Respiratory Panel and the Blood Culture Identification Panel (both of which are CE-marked and FDA-cleared) and the GI panel which is FDA cleared. BioFire anticipates CE IVD marking of the FilmArray ${ }^{\circledR}$ GI Panel in late spring of 2014 [11]. Approval from Philippine FDA was given last June 2015. 
In October 2015, the U.S. Food and Drug Administration (FDA) approved the FilmArray Meningitis/Encephalitis (ME) panel (BioFire Diagnostics, Salt Lake City, UT), the first multiplex PCR panel for detection of CNS infections [12].

\section{Methods}

In this descriptive, observational study, a review of records of those who were tested with FilmArray Gastrointestinal, Blood Culture Identification, Meningitis Encephalitis, and Respiratory Panels in the Section of Microbiology and Cellular Immunology, Institute of Pathology of St. Luke's Medical Center - Global City from October 2017 to September 2019. All those who were tested for FilmArray Panels during the inclusive period were included in the study, whether inpatient or outpatient. Excluded were data of those who had invalid or equivocal results as well as those who tested more than once during an admission, only the first test was included in the the study. Database of the Section of Microbiology and Cellular Immunology were searched for those who were tested for Gastrointestinal, Blood Culture Identification, Meningitis Encephalitis, and Respiratory Panels during the study period. Each test result was assigned a code and the data collection form was filled up whether it was an outpatient or an inpatient request, type of sample submitted, age and gender of the patient, FilmArray panel done, the result of the test, and the organism/s detected, if any. The proportion of the organisms detected in the positive tests of the different FilmArray panels was determined. In cases of multiple pathogens detected, the proportion of bacterial with parasitic, bacterial with fungal, bacterial with viral, or any combination hereof was determined.

Data gathered were analyzed using percentage and proportion represented in tables and charts. Proportion of the inpatient and outpatient test requests, proportion of the different type of samples received for each film array panel, and the proportion of the positive as well as the negative tests for each FilmArray panel were divided by the number of tests received for the two years study period for each FilmArray panel. The distribution of the different organisms detected were divided by the number of positive tests received for the two years study period for each FilmArray panel. The distribution of requesting physicians were divided using the total number of tests requested for each panel as denominator.

\section{Results}

A total of 547 tests were included for the two-year period covered by the study. Majority of the patients tested were from those aged 60 years old and above at $29.8 \%$. Both the Gastrointestinal and BCID panels were also requested to this age group. The Respiratory panel was requested mostly to the pediatric age group aged 1 year old and below. The Meningitis Encephalitis Panel was mostly requested among those who are between 19 to 39 years old. Patients who had the panels tested were mostly males except for the Respiratory panel which had female predominance. These panels were mostly requested among inpatients at $69 \%$. Although some outpatients are actually send-outs from patients admitted in other hospitals. Table 1 below summarizes the demographics of the patients who were included in the study.

The most requested FilmArray panel was the Gastrointestinal panel with 261 requests ( $48 \%$ ), followed by the Meningitis/ Encephalitis Panel with 143 tests (26\%), then Respiratory panel with 125 tests (23\%), and lastly, by the Blood Culture Identification Panel with 18 tests $(3 \%)$ in the span of two years. This is shown in Figure 1 below.

Majority of the samples submitted for the Gastrointestinal panel are stool samples with only one colonic aspirate submitted. Most of the samples submitted for Respiratory Panel are nasopharyngeal swabs (85). The rest are nasal swabs (37), oropharyngeal swabs (2), and a tracheal aspirate. Samples submitted for Meningitis Encephalitis Panel and Blood Culture identification Panel are cerebrospinal fluid and blood from blood culture bottles which flagged positive from the blood culture machine, respectively. The positivity rate of each Panel are shown in Table 2 below.

Table 1: Demographics of the Patients who were tested with the four Biofire FilmArray Multiplex PCR Panels at St. Luke's Medical Center - Global City from October 2017 to September 2019

\begin{tabular}{|c|c|c|c|c|c|c|c|c|c|c|}
\hline \multirow{2}{*}{ Category } & GI & Panel & Respiratory & Panel & ME & Panel & BCID & Panel & \multirow{2}{*}{ Total } & \multirow{2}{*}{$\%$} \\
\hline & $\mathrm{n}$ & $\%$ & $n$ & $\%$ & $n$ & $\%$ & $\mathrm{n}$ & $\%$ & & \\
\hline \multicolumn{11}{|l|}{ Age (years) } \\
\hline$<o r=1$ & 13 & 5 & 33 & 26.4 & 5 & 3.5 & 2 & 11 & 53 & 9.7 \\
\hline 02-May & 8 & 3.1 & 18 & 14.4 & 3 & 2 & - & - & 29 & 5.3 \\
\hline 06-Oct & 3 & 1.1 & 13 & 10.4 & 2 & 1.4 & 1 & 5.5 & 19 & 3.5 \\
\hline Nov-18 & 2 & 0.8 & 11 & 8.8 & 5 & 3.5 & - & - & 18 & 3.3 \\
\hline 19- 39 & 58 & 22.2 & 17 & 13.6 & 57 & 39.9 & 1 & 5.5 & 133 & 24.3 \\
\hline $40-59$ & 70 & 26.8 & 19 & 15.2 & 39 & 27.3 & 4 & 22.2 & 132 & 24.1 \\
\hline$>$ or $=60$ & 107 & 41 & 14 & 11.2 & 32 & 22.4 & 10 & 55.6 & 163 & 29.8 \\
\hline Total & 261 & 100 & 125 & 100 & 143 & 100 & 18 & 100 & 547 & 100 \\
\hline \multicolumn{11}{|l|}{ Gender } \\
\hline Male & 143 & 55 & 58 & 46 & 88 & 62 & 11 & 61 & 300 & 55 \\
\hline Female & 118 & 45 & 67 & 54 & 55 & 38 & 7 & 39 & 247 & 45 \\
\hline TOTAL & 261 & 100 & 125 & 100 & 143 & 100 & 18 & 100 & 547 & 100 \\
\hline \multicolumn{11}{|c|}{ Type of patient care } \\
\hline Inpatient & 168 & 64 & 98 & 78 & 96 & 67 & 18 & 100 & 380 & 69 \\
\hline Outpatient & 93 & 36 & 27 & 22 & 47 & 33 & - & - & 167 & 31 \\
\hline Total & 261 & 100 & 125 & 100 & 143 & 100 & 18 & 100 & 547 & 100 \\
\hline & & & & & & & & & & 003 \\
\hline
\end{tabular}

Citation: Lagamayo EN, Rusia-Uy RO (2021) A Two-year review of the use of film array in a tertiary hospital in the Philippines. Arch Pulmonol Respir Care 7(1): 001- 
Of the 156 Gastrointestinal Panel tests which yielded positive test results, majority had single pathogens identified either a bacteria or a virus at $52.6 \%$. The rest of the tests yielded a combination of bacterial, viral, and or parasitic etiologies. These are summarized in the succeeding Table 3.

Of the 71 positive tests of the Respiratory Panel, majority also detected single pathogen at $97.18 \%$ with only 2 tests showing multiple viral pathogens. These are represented in Table 4 .

Of the 20 tests which turned out positive in the Meningitis Encephalitis Panel, only one yielded multiple pathogens, while the rest yielded a single bacterial, viral, or fungal pathogen. These are shown in Table 5.

All of the 15 positive Blood Culture Identification Panels in Table 6 below yielded single pathogen and some detected resistance genes Identification Panels done at St. Luke's Medical Center - Global City from October 2017 to September 2019.

As noted in Table 7, most of the requesting physicians were specialists of internal medicine with subspecialties corresponding to the panels requested. For example gastroenterologists were the most requesting physicians for GI Panel. Subsequently, most of the ME panels were requested by Neurologists. It was noted in previous tables that most of the respiratory panels were requested among the pediatric age group, thus, this panel is mostly requested by Pediatricians. The Blood Culture Identification panels were mostly requested by Pulmonologists as well as other Adult medicine physicians and subspecialists.

\section{Discussion}

Multiplex PCR panels are a new technology for rapid diagnosis of infectious diseases. This study described the usage of the Multiplex PCR System (FilmArray ${ }^{\circledR}$ ) for diagnosing suspected blood-borne, CNS, gastrointestinal, and respiratory

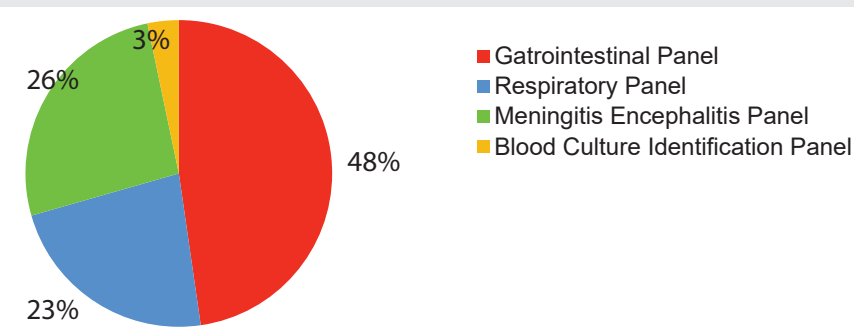

Figure 1: Proportion of the four Biofire FilmArray Panels done at St. Luke's Medical Center - Global City from October 2017 to September 2019

Table 2: Positivity rate of the Biofire FilmArray Panels done at St. Luke's Medical Center - Global City from October 2017 to September 2019.

\begin{tabular}{|c|c|c|c|c|}
\hline TEST & Positive & Negative & TOTAL & Positivity Rate (\%) \\
\hline GI Panel & 156 & 105 & 261 & 60 \\
\hline Respiratory Panel & 71 & 54 & 125 & 57 \\
\hline ME Panel & 20 & 123 & 143 & 14 \\
\hline BCID & 15 & 3 & 18 & 83 \\
\hline
\end{tabular}

Table 3: List of the Organisms Detected and Their Frequencies in the Gastrointestinal Panels done at St. Luke's Medical Center - Global City from October 2017 to September 2019.

\begin{tabular}{|c|c|c|}
\hline Organisms & $\mathbf{n}$ & $\%$ \\
\hline Bacteria (single pathogen) & \multirow{3}{*}{28} & \multirow{3}{*}{17.9} \\
\hline Escherichia coli (EAEC,EIEC, ETEC, & & \\
\hline STEC,EPEC, 0157) & & \\
\hline Clostridium difficile & 12 & 7.7 \\
\hline Cyclospora cayetanensis & 11 & 7.1 \\
\hline Salmonella & 10 & 6.4 \\
\hline Campylobacter & 7 & 4.5 \\
\hline Vibrio & 2 & 1.3 \\
\hline Vibrio cholerae & 2 & 1.3 \\
\hline Plesiomonas shigelloides & 1 & 0.6 \\
\hline \multicolumn{3}{|l|}{ Virus (single pathogen) } \\
\hline Norovirus GI/GII & 4 & 2.6 \\
\hline Rotavirus A & 2 & 1.3 \\
\hline Adenovirus F 40/41 & 2 & 1.3 \\
\hline Astrovirus & 1 & 0.6 \\
\hline \multicolumn{3}{|l|}{ Multiple pathogens } \\
\hline Two or more bacterial pathogen $(2-6)$ & 46 & 29.5 \\
\hline Bacterial and viral pathogens (2-5) & 18 & 11.5 \\
\hline Bacterial and parasitic pathogens $(2-3)$ & 9 & 5.8 \\
\hline Two or more viral pathogens & 1 & 0.6 \\
\hline Total & 156 & 100 \\
\hline
\end{tabular}

Table 4: List of the Organisms Detected and Their Frequencies in the Respiratory Panels done at St. Luke's Medical Center - Global City from October 2017 to September 2019.

\begin{tabular}{|c|c|c|}
\hline Organisms & $\mathbf{n}$ & $\%$ \\
\hline Single Pathogen & & \\
\hline Influenza A (H1, H3, H1-2009) & 17 & 23.94 \\
\hline Human Rhinovirus/ Enterovirus & 14 & 19.72 \\
\hline Influenza B & 12 & 16.9 \\
\hline Respiratory Syncytial Virus & 8 & 11.27 \\
\hline Human Metapneumovirus & 6 & 8.45 \\
\hline Adenovirus & 5 & 7.04 \\
\hline Parainfluenza (1,2,3,4) & 4 & 5.63 \\
\hline Bordetella pertussis & 2 & 2.82 \\
\hline Coronavirus (HKU, NL63, 229E, OC43) & 1 & 1.41 \\
\hline Multiple Pathogens & 1 & 1.41 \\
\hline Adenovirus and Coronavirus & 71 & 1.41 \\
\hline Adenovirus, Coronavirus, and Influenza A & 100 \\
\hline Total & & \\
\hline & & 1 \\
\hline
\end{tabular}

infections done in the Institute of Pathology, St. Luke's Medical Center - Global City from October 2017 to September 2019.

The mostly used among all four panels is the Gastrointestinal Panel which comprised $48 \%$ of the total 547 FilmArray panel tests done during the two-year period.

These test panels were requested to patients of all age groups, from pediatrics less than a year old to more than a 100 years old. The various panels were requested from different age groups. The Gastrointestinal and the Blood Culture Identification Panels which are mostly requested among patients who are 60 years old and older. The respiratory panel was mostly requested among those who are one year old 
and younger. The Meningitis Encephalitis panel was mostly requested among patients between 19 and 39 years of age. These panels are requested mostly in males except for for the respiratory panel which was requested predominantly among female patients. All four panels were requested more on inpatients rather than outpatients. The specimen submitted for the each panel was almost uniform except for the respiratory panel which had four different sample types: nasopharyngeal swab, nasal swab, oropharyngeal swab, and tracheal aspirate.

Table 5: List of the Organisms Detected and Their Frequencies in the Meningitis Encephalitis Panels done at St. Luke's Medical Center - Global City from October 2017 to September 2019.

\begin{tabular}{|c|c|c|}
\hline Organisms & $\mathbf{n}$ & $\%$ \\
\hline Single Pathogen & & 20 \\
\hline Streptococcus pneumoniae & 4 & 10 \\
\hline Escherichia coli & 2 & 10 \\
\hline Enterovirus & 2 & 10 \\
\hline Varicella Zoster Virus & 2 & 10 \\
\hline Cytomegalovirus & 2 & 10 \\
\hline Human Herpes Virus 6 (HHV6) & 2 & 10 \\
\hline Cryptococcus neoformans & 2 & 10 \\
\hline Herpes Simplex Virus 1 & 2 & 5 \\
\hline Herpes Simplex Virus 2 & 1 & 5 \\
\hline Multiple Pathogens & 1 & 100 \\
\hline Neisseria meningitidis and HHV 6 & 20 & \\
\hline Total & & \\
\hline
\end{tabular}

Table 6: List of the Organisms Detected and Their Frequencies in the Blood Culture.

\begin{tabular}{|c|c|c|}
\hline Analyte & $\mathbf{n}$ & $\%$ \\
\hline Single Pathogen & & 46 \\
\hline Staphylococcus, mec A gene & 7 & 7 \\
\hline Neisseria meningitidis & 1 & 7 \\
\hline Enterobacteriaceae (Klebsiella) & 1 & 13 \\
\hline Staphylococcus aureus & 2 & 20 \\
\hline Enterobacteriaceae (Escherichia coli) & 3 & 7 \\
\hline Enterobacteriacieae & 1 & 100 \\
\hline
\end{tabular}

The positivity rates of the Gastrointestinal, Respiratory, and Blood Culture Identification panels were also high at $60 \%$, $57 \%$, and $83 \%$, respectively. The Gastrointestinal Panel yielded $5.8 \%$ single viral pathogen which may have been missed by Stool Bacterial Culture. A high percentage (97\%) of detected organisms in the Respiratory panel are viruses which could have been missed in bacterial cultures. The lowest positivity rate was seen with the Meningitis Encephalitis Panel at $14 \%$. Despite a low positivity rate, most of the detected pathogens were viruses which may not have been grown in the usual CSF bacterial culture. This panel had only one codetection which is the presence of both Neisseria meningitidis and Human Herpes Virus 6. The single pathogens detected bacterial, viral, or fungal etiology.

All 15 positive Blood Culture Identification Panels detected a single bacterial pathogen and some with resistance genes. The most commonly detected organism was Staphylococcus with mecA resistance gene at $46 \%$. The negative blood cultures were reviewed and these showed growth of anaerobic organisms or pathogens which were not included in the Blood Culture Identification Panel. Having known the presence of these resistance genes would aid the clinicians in their choice of antimicrobials.

A variety of doctors request the four panels: Gastroenterologists, Pulmonologists, neurologists, Infectious Disease Specialists, Surgeons, other adult medicine specialists, Pediatricians, Pediatric pulmonologists, Pediatric Neurologists, Pediatric Gastroenterologists, Pediatric Infectious Disease Specialists, Neonatologists, and other Pediatric specialists. The most common requesting physicians are the Gastroenterologists (25.9\%) followed by Infectious Disease Specialists (15.9\%). Collectively, Pediatricians and other Pediatric Specialty Physician comprise $19.5 \%$ of the requesting doctors. There are those with no known requesting physicians which are mostly from the outpatient care service and those which were sendouts from other hospitals (14.1\%).

There is a study in the diagnostic modalities used in the diagnosis of sepsis and it mentioned that, "The main disadvantages of analyzing specimens after they have grown in culture are the delay in time and the potential bias introduced

Table 7: List of the Requesting Physicians of the Biofire FilmArray Panels done at St. Luke's Medical Center - Global City from October 2017 to September 2019.

\begin{tabular}{|c|c|c|c|c|c|c|c|c|c|c|}
\hline Requesting & GI & \multirow{2}{*}{$\%$} & Respiratory & \multirow{2}{*}{$\%$} & ME & \multirow{2}{*}{$\%$} & BCID & \multirow{2}{*}{$\%$} & \multirow{2}{*}{ Total } & \multirow{2}{*}{$\%$} \\
\hline Physicians & Panel & & Panel & & Panel & & Panel & & & \\
\hline Gastro & 142 & 54.4 & - & - & - & - & - & - & 142 & 26 \\
\hline ID & 38 & 14.6 & 22 & 17.6 & 23 & 16.1 & 4 & 22.2 & 87 & 15.9 \\
\hline Pulmo & 14 & 5.4 & 8 & 6.4 & 4 & 2.8 & 5 & 27.8 & 31 & 5.7 \\
\hline Neuro & 1 & 0.4 & - & - & 48 & 33.5 & - & - & 49 & 8.9 \\
\hline Others-Adult & 31 & 11.9 & 7 & 5.6 & 11 & 7.7 & 5 & 27.8 & 54 & 9.9 \\
\hline Pedia & 7 & 2.7 & 27 & 21.6 & 1 & 0.7 & 1 & 5.55 & 36 & 6.6 \\
\hline Ped Pulmo & 2 & 0.8 & 18 & 14.4 & 1 & 0.7 & - & - & 21 & 3.8 \\
\hline Ped Neonat & 4 & 1.5 & 6 & 4.8 & 1 & 0.7 & - & - & 11 & 2 \\
\hline Ped Gastro & 3 & 1.1 & - & - & - & - & - & - & 3 & 0.5 \\
\hline Ped ID & 9 & 3.4 & 12 & 9.6 & 3 & 2.1 & - & - & 24 & 4.4 \\
\hline Ped Neuro & - & - & 1 & 0.8 & 5 & 3.5 & 2 & 11.1 & 8 & 1.5 \\
\hline Others - Ped & - & - & 4 & 3.2 & - & - & - & - & 4 & 0.7 \\
\hline None & 10 & 3.8 & 20 & 16 & 46 & 32.2 & 1 & 5.55 & 77 & 14.1 \\
\hline Total & 261 & 100 & 125 & 100 & 143 & 100 & 18 & 100 & 547 & 100 \\
\hline
\end{tabular}

Citation: Lagamayo EN, Rusia-Uy RO (2021) A Two-year review of the use of film array in a tertiary hospital in the Philippines. Arch Pulmonol Respir Care 7(1): 001- 
by culture steps. Furthermore, uncultivable organisms cannot be identified by these techniques [2]."

There are several limitations of this study including the fact that this is a retrospective design done in a single hospital setting and done at a time when the tests were first introduced. The FilmArray results were also not compared with the conventional culture methods done, if any. This study could be extended up to such time when the clinicians are already aware of the tests. The study can also be done to compare the results of the FilmArray and the conventional culture.

The authors have no conflict of interest with the manufacturers of FilmArray that may have influenced either the conduct or the presentation of the research.

\section{Conclusion}

The Multiplex PCR FilmArray Panels were useful both in the outpatient and inpatient care. These can be used in a wide range of patients and some may be used using different sample types like the Respiratory Panel. This is probably the reason why these panels are also requested by physicians both in Adult and Pediatric Medicine. The high positivity rates (more than $50 \%$ in the Blood Culture Identification Panel, Respiratory, and Gastrointestinal Panels) provide early patient diagnosis. In turn, requesting physicians can then allow early administration of appropriate targeted therapy and management especially with the detection of resistance genes. Another critical role it plays is the identification of causative pathogens which may have been missed by traditional culture methods such as fastidious organisms, viruses, and parasites, thereby reducing the unnecessary use of antimicrobials in some of these cases and greatly help in the reduction of the development of antimicrobial resistance.

\section{References}

1. WHO (2018) Sepsis. Link: https://bit.ly/39GRK30

2. Liesenfeld O, Lehman L, Hunfeld KP, Kost G (2014) Molecular diagnosis of sepsis: New aspects and recent developments. European Journal of Microbiology Immunology 4: 1-25. Link: https://bit.ly/3cxOWsH

3. WHO (2018) Antimicrobial resistance. Link: https://bit.ly/3rd6i05

4. Tsalik EL, Jones D, Nicholson B, Waring L, Liesenfeld O, et al. (2010) Multiplex PCR To Diagnose Bloodstream Infections in Patients Admitted from the Emergency Department with Sepsis. J Clin Microbiol 48: 26-33. Link: https://bit.ly/2MuOi2y

5. AACC (2015) Use of Multiplex PCR for Diagnosis of Infectious Syndromes Link: https://bit.ly/3jhkZg8

6. Javali M, Acharya P, Mehta A, John AA, Mahale R, et al. (2017) Use of multiplex PCR based molecular diagnostics in diagnosis of suspected CNS infections in tertiary care setting-A retrospective study. Clinical Neurology Neurosurgery 161: 110-116. Link: https://bit.ly/3pGMP7D

7. Elnifro EM, Ashshi AM, Cooper RJ, Klapper PE (2000) Multiplex PCR: Optimization and Application in Diagnostic Virology. Clinical Microbiology Reviews 13: 559-570. Link: https://bit.ly/202r1pr

8. Giordano G, Garzon M, Velaro M, Abiega C, Zarate A, et al. (2018) Preliminary experience with the use of Multiplex PCR System (FilmArray®) in a hospital from Argentina. International Journal in Infectious Diseases 73: 169-170. Link: https://bit.ly/3j7F5tb

9. BIOFIRE® FILMARRAY® SYSTEMS 2019. Link: https://bit.ly/3j8fCzE

10. BIOFIRE® FILMARRAY® Multiplex Real-Time PCR Systems 2019 Link: Link: https://bit.ly/39FHGqX

11. BioFire's FilmArray ${ }^{\circledR}$ Gastrointestinal Panel Receives FDA Clearance. Link: https://bit.ly/3tkfrpo

12. Radmard S, Reid S, Ciryam P, Boubour A, Ho N, et al. (2019) Clinical Utilization of the FilmArray Meningitis/Encephalitis (ME) Multiplex Polymerase Chain Reaction (PCR) Assay. Front Neurol 10: 281. Link: https://bit.ly/2NUyTZP
Discover a bigger Impact and Visibility of your article publication with

\section{Peertechz Publications}

\section{Highlights}

* Signatory publisher of ORCID

* Signatory Publisher of DORA (San Francisco Declaration on Research Assessment)

* Articles archived in worlds' renowned service providers such as Portico, CNKI, AGRIS, TDNet, Base (Bielefeld University Library), CrossRef, Scilit, J-Gate etc.

* Journals indexed in ICMJE, SHERPA/ROMEO, Google Scholar etc.

* OAI-PMH (Open Archives Initiative Protocol for Metadata Harvesting)

* Dedicated Editorial Board for every journal

* Accurate and rapid peer-review process

* Increased citations of published articles through promotions

- Reduced timeline for article publication

Submit your articles and experience a new surge in publication services (https://www.peertechz.com/submission).

Peertechz journals wishes everlasting success in your every endeavours.

Copyright: @ 2021 Lagamayo EN, et al. This is an open-access article distributed under the terms of the Creative Commons Attribution License, which permits unrestricted use, distribution, and reproduction in any medium, provided the original author and source are credited. 